\title{
A software system for the collection of retrospective protocols prompted by eye fixations
}

\author{
J. EDWARD RUSSO \\ University of Chicago, Chicago, Illinois 60637
}

\begin{abstract}
A software system is capable of recording eye position, reducing these data to a sequence of eye fixations, and immediately replaying this fixation sequence superimposed over the original stimulus. The replay can be controlled by the subject to produce various speeds, to pause, or to back up to earlier fixations. The purpose of the replay software is to enable the eye fixations to prompt a retrospective verbal protocol. Such a prompted protocol should reduce the forgetting and fabrication problems that are often associated with retrospective protocols. In a preliminary experimental test, the prompted protocol method yielded longer protocols and contained more words than those generated by either the concurrent or retrospective protocol methods.
\end{abstract}

A software system capable of immediate replay of recorded eye fixations is designed to solve a problem associated with verbal protocols. The generation of concurrent "thinking aloud" protocols may interfere with the performance of the primary task. Eliminating this interference by collecting the protocol retrospectively introduces other problems: Much of the processing may not be recallable or, worse yet, may be fabricated. A possible solution to this predicament is the use of a retrospective protocol prompted by a replay of the recorded eye fixations.

This paper describes a software system for collecting such prompted protocols. It is divided into four major sections: the recording and on-line analysis of eye fixations, the replay software and its control, its use to record prompted protocols, and some preliminary results of the usefulness of prompting a protocol with eye fixations.

\section{RECORDING AND ON-LINE ANALYSIS OF EYE FIXATIONS}

To maximize the prompting efficacy of the eye fixations, they should be presented to subjects as soon as possible after task completion. This necessitates on-line analysis of the raw eye positions into a sequence of fixations.

The collection of a sequence of eye fixations begins with a device to sense eye position. I am presently using a commercial corneal reflectance apparatus (Model 1996, Applied Science Laboratories, Gulf \& Western) and have also used a homemade limbus reflection sensor (Russo, 1975; Russo \& Mathews, Note 2). Many other devices are acceptable, the prerequisite being that the sensed position be processible by computer. This rules out video recording techniques whose records must be coded by an experimenter. (For a review of methods, refer to Young and Sheena, 1975.)

The analysis software must reduce the stream of eye positions (horizontal and vertical coordinates) to a sequence of fixations. Each fixation must be identified by its rank order, location, and duration. Various algorithms for identifying when the eye is fixed are in use. These include criteria based on zero velocity (Reder, 1973), on a delimited area for a sequence of sensed positions, and location within calibrated boundaries (Goode \& Russo, 1970). The software described here uses the second criterion.

As eye positions are collected, the analysis software fills a table with the order, location, and duration of easy eye fixation. When a subject's buttonpress signals task completion, this table is terminated and made available to the REPLAY program.

\section{REPLAY WITH INTERACTIVE CONTROL}

The goal of the REPLAY software is to superimpose the sequence of eye fixations on the original stimulus. For example, Russo and Rosen (1975) used a CRT to present verbal descriptions of six used cars. Subjects chose their most preferred alternative, requiring an average of $30 \mathrm{sec}$ to make that choice. A few seconds after the subject's buttonpress ended the trial and erased the stimulus, a ready signal appeared on the CRT. When the subject was prepared to speak into a microphone, a buttonpress activated the REPLAY software.

This software paints on the CRT the order and duration of the first fixation beside that part of the stimulus that was fixated, for example, beside the used car that was looked at first. REPLAY proceeds to the second fixation, painting the numeral " 2 " and the 
fixation duration beside the stimulus component fixated second, and so forth.

The most critical factor in the control of the replay of eye fixations is speed. The replay must proceed at a rate compatible with recall and speaking. The REPLAY routine can present the fixation sequence at actual speed or slow it down by factors of two, four, or eight. It can also increase the speed to two and four times the original rate. The replay speed is controlled by the subject through a gimbal, that is, by moving a potentiometer whose signal is converted to digital values. The current replay speed is displayed for the subject near the center of the CRT. The slower speeds are used most frequently. It is essentially impossible to generate the prompted protocol as quickly as the original task was completed.

Besides the speed control, the top of the gimbal holds a button that can cause the replay program to pause at the current fixation. This enables the subject to speak at some length about a particular fixation or, more often, about an episode of several fixations that has just been replayed.

Finally, a back-up mode is available. If the gimbal is pulled toward the subject from the vertical position, the REPLAY software backs up to earlier fixations at rates of $1 / 8,1 / 4,1 / 2,1,2$, and 4 times normal speed. The back-up option is seldom exercised. When subjects do use it, they usually return only a few fixations. This REPLAY software is similar to an earlier version described by Conery, Smith, and Russo (1975).

\section{PROMPTED PROTOCOLS}

The process tracing methodologies of eye fixations and verbal protocols are complementary (Russo, 1978a). The weak points of one method tend to be compensated by the advantages of the other. From the viewpoint of the psychologist recording eye fixations, the problem is how to interpret this detailed sequence of information acquisition responses. This interpretation is often far from transparent. Either our theories make no predictions about these data, or the eye fixations themselves are only the tip of the iceberg of cognitive processing. There is even a problem with matching cognitive processing with the observed fixation duration; the two need not be coterminous (Russo, 1978b). For all the above reasons, a verbal protocol in which the subjects describe their ongoing thoughts can provide essential help in interpreting the fixation data.

Ideally, the verbal protocol should be collected concurrently with task performance. However, the simultaneous performance of a second task, generating a verbal protocol, may interfere with performance of the task of primary research interest. Certainly task performance is altered in superficial ways (e.g., by requiring longer to complete it), and it may be changed more fundamentally (although there is no evidence demonstrating this). In addition to concerns about validity, there are practical problems. Many eye position sensors require that the head be fixed, for example, by a bite bar or chin rest. These restraints usually prohibit speaking. For both reasons, one turns to retrospective protocols.

The problems here are forgetting and fabrication. The longer the time required to complete the primary task, the less processing detail can be recalled. If the task takes more than a minute to complete, it is very likely that important information will not be recalled. The problem of fabrication, however, is more insidious. There is a well-documented tendency for people to reconstruct the details of past events based on higher level concepts, expectations, or sterotypes (e.g., D'Andrade, 1974). The danger of this in retrospective verbal protocols is delineated by Nisbett and Wilson (1977) and Smith and Miller (1978). For a comprehensive discussion of this type of protocol, see Ericsson and Simon (Note 1).

A possible technique for reducing both problems is prompting the retrospective protocol by the eye fixation sequence. The fixations cue memory and thereby reduce forgetting. Indeed, they go too far; subjects are usually not able to account for every eye fixation. Prompting should also reduce reconstructive invalidity since the reconstruction must, at a minimum, conform to the actual fixation sequence. Ideally, the effectiveness of the fixations as prompts would reduce the need for any reconstruction by cuing a recall of the thoughts that actually occurred. Of course, there is no guarantee of this, and we can expect some reconstruction to occur even with a valid and detailed prompt like the fixation sequence.

It might be useful to present the instructions to subjects who generated prompted protocols. The subjects were first taught to produce a concurrent protocol, then a retrospective protocol, and finally they were read the following:

"This procedure for describing how you solve a problem is very similar to the last one. You will perform the task silently, and then tell us what you were thinking. The difference is that you will have your own eye fixations, superimposed on the original stimulus. We want you to tell us what you were thinking during every eye fixation. We want you to explain to us why you looked where you looked and what you were thinking when you looked there.

"The replay of the eye fixations will be completely under your control. You can make it go as fast or as slow as you wish. You can stop it at any time. You can also back it up if you wish. Please try to remember as best you can what happened during every eye fixation. If you cannot remember, please say so. If several eye fixations go by without your explaining what you were thinking, we will remind you by saying: "Please tell us what you were thinking during that eye fixation."'

\section{EXPERIMENT}

An initial experimental comparison of the three protocol techniques has been conducted. The results 
have not been fully analyzed, but a preliminary report of the major findings is possible.

Six subjects performed each of the three methods (concurrent, retrospective, and prompted) for one of three tasks in a Latin-square design. The three tasks were anagrams, choice between two simple gambles, and Raven's progressive matrices. They were chosen to represent verbal, numerical, and pictorial problem-solving tasks, respectively. A Latin-square design was used because of possible carry-over effects between methods on the same task. Fifteen trials were performed in each task.

The primary summary statistics from the protocols are reported in Table 1. The prompted protocols were considerably longer than concurrent or retrospective protocols. Of the latter two methods, concurrent protocols included the time to both generate the protocol and perform the primary task, while the retrospective method included only the time to generate a protocol. Thus, concurrent protocols should take longer even if they produced no more words. This expectation was confirmed when the number of words spoken was tabulated. Again, the prompted method delivered a longer protocol, but the difference between concurrent and retrospective protocols vanished. Both yielded around 94 words per problem, compared to the 238 produced by prompted protocols.

These preliminary results are encouraging. They support the prediction that protocols prompted by eye fixations will be superior to the two existing methods. However, the only superiority that has been demonstrated is in quantity, not quality. Prompted protocols are longer, in time and number of words, but the value of the additional words has not been demonstrated. It is expected that coding the protocols for specific "informative" statements will better test the potential value of the prompted method.

\section{CONCLUSION}

Eye fixations have been used to produce a new type of verbal protocol. This is an example of the interactive, rather than parallel, use of two different process tracing methodologies. That is, this use generates observations that could not be obtained from the simultaneous but independent use of both methods. For example, if a verbal protocol and eye fixations are collected concurrently, the former would be no longer than the usual concurrent protocol and would not be linked to the fixation sequence in ways that might provide critical insight. The availability of laboratory computers may
Table 1

Length of Protocols Generated by Each Method

\begin{tabular}{cccc}
\hline & \multicolumn{3}{c}{ Method } \\
\cline { 2 - 4 } Measure & $\begin{array}{c}\text { Con- } \\
\text { current }\end{array}$ & $\begin{array}{c}\text { Retro- } \\
\text { spective }\end{array}$ & Prompted \\
\hline Total Time (sec) & 62.9 & 40.4 & 121.7 \\
Number of Words & 95.9 & 92.4 & 237.9 \\
\hline
\end{tabular}

enable the development of more interactive methodologies, whose benefits are greater than the sum of their parts.

\section{REFERENCE NOTES}

1. Ericsson, K. A., \& Simon, H. A. Retrospective verbal reports as data. C.I.P. Working Paper No. 388, Department of Psychology, Carnegie-Mellon University, August 4, 1978.

2. Russo, J. E., \& Mathews, P. Tracking eye fixations in cognitive tasks. Unpublished manuscript, June 1975.

\section{REFERENCES}

Conery, J. S., Smith, R. T., \& Russo, J. E. Interactive computer techniques. Proceedings of the Digital Equipment Users Society, 1975, 1, 389-391.

D'ANDrade, R. G. Memory and the assessment of behavior. In H. M. Blalock, Jr. (Ed.), Measurement in the social sciences. Chicago: Aldine, 1974.

Goode, F. M., \& Russo, J. A LINC-8 software system for the recording and on-line analysis of eye movements. Proceedings of the 78th Annual Convention of the American Psychological Association, $1970,845.846$.

Nisbett, R. E., \& Wilson, T. D. Telling more than we can know: Verbal reports on mental processes. Psychological Review, 1977, 84, 231-259.

REDER, S. M. On-line monitoring of eye-position signals in contingent and noncontingent paradigms. Behavior Research Methods \& Instrumentation, 1973, 5, 218-228.

Russo, J. E. The limbus reflection method for measuring eye position. Behavior Research Methods \& Instrumentation, 1975, 7, 205-208.

Russo, J. E. Eye fixations can save the world: A critical evaluation and a comparison with other information processing methodologies. In H. K. Hunt (Ed.), Advances in consumer research (Vol. 5). Ann Arbor, Mich: Association for Consumer Research, 1978. Pp. 561-570. (a)

Russo, J. E. Adaptation of cognitive processes to the eye movement system. In J. W. Senders, D. F. Fisher, \& R. A. Monty (Eds.), Eye movements and the higher psychological functions. Hillsdale, N.J: Erlbaum, 1978. (b)

Russo, J. E., \& Rosen, L. D. An eye movement analysis of multialternative choice. Memory \& Cognition, 1975, 3, 267-276.

SMrth, E. R., \& Miller, F. D. Limits on perception of cognitive processes: A reply to Nisbett and Wilson. Psychological Review, $1978,85,355-362$.

Young, L. R., \& SheEna, D. Survey of eye movement recording methods. Behavior Research Methods \& Instrumentation, 1975, 7, 397-429. 